\title{
Prevalence of hypercalcemia of malignancy among pediatric cancer patients in the UK Clinical Practice Research Datalink database
}

This article was published in the following Dove Press journal:

Clinical Epidemiology

I5 June 2017

Number of times this article has been viewed

\author{
Susan Jick' \\ Lin $\mathrm{Li}^{\prime}$ \\ Victor M Gastanaga ${ }^{2}$ \\ Alexander Liede ${ }^{2}$ \\ Rohini K Hernandez ${ }^{2}$ \\ 'Boston Collaborative Drug \\ Surveillance Program, Boston \\ University School of Public Health, \\ Lexington, MA, USA; ${ }^{2}$ Center for \\ Observational Research, Amgen \\ Inc., Thousand Oaks and South San \\ Francisco, CA, USA
}

Correspondence: Susan Jick

Boston Collaborative Drug Surveillance Program, Boston University School of Public Health, II Muzzey Street, Lexington, MA 0242I, USA

Tel + I 78I 8626660

Fax +I 78I 8621680

Email sjick@bu.edu
Background: The reported proportion of cancer patients who experience hypercalcemia of malignancy (HCM) is low, particularly in the pediatric population, ranging between $<1 \%$ and $5 \%$. HCM can be observed with any type of tumor in children and occurs most commonly with leukemia. While HCM is a potentially fatal condition, the prevalence of HCM is not well understood in pediatric cancer patients.

Methods: Using the UK Clinical Practice Research Datalink, we identified pediatric cancer patients with recorded corrected serum calcium (CSC) from 2003 through 2014. Hypercalcemic patients (CSC $\geq 10.8 \mathrm{mg} / \mathrm{dL}$ ) were classified into 4 CSC levels. We estimated the annual prevalence of HCM using Byar's method.

Results: Among 517 pediatric cancer patients, leukemia, lymphoma, and brain tumors were the most frequent cancer types. The prevalence of HCM overall (grade 1 or higher) ranged from $0.24 \%$ to $0.81 \%$ between 2003 and 2014 . There were too few cases to compare prevalence by type of cancer.

Conclusion: We provide the first systematic analysis using a UK population-based data source to estimate the number of pediatric cancer patients affected with HCM by grade. Our findings showed that the prevalence of pediatric HCM was very low $(0.24 \%-0.81 \%)$ over the 12 -year study period, which is consistent with previous study of adult cancer patients in the UK $(0.20 \%-0.67 \%)$.

Keywords: hypercalcemia, pediatric, cancer, prevalence, Clinical Practice Research Datalink

\section{Plain language summary}

Hypercalcemia of malignancy (HCM) occurs among people with cancer and is the result of bone resorption, which leads to raised calcium levels in the blood. The reported proportion of cancer patients who experience HCM is low, particularly in a pediatric population, ranging between $<1 \%$ and $5 \%$. HCM can be observed with any type of cancer but occurs most commonly in children with leukemia. While HCM can lead to death, we do not know what proportion of pediatric cancer patients have this condition. We conducted this study to estimate the proportion of HCM in children in the UK's Clinical Practice Research Datalink. We identified all children in the database from 2003 through 2014 who had a cancer diagnosis. From this population, we identified those who developed HCM and then estimated the HCM prevalence rates. The prevalence of pediatric HCM was very low over the 12-year study period (ranging from $0.24 \%$ to $0.81 \%$ ).

\section{Introduction}

Hypercalcemia is a condition defined by a serum calcium level above the upper limit of the normal reference range. When hypercalcemia occurs in cancer patients 
during the course of the disease, it is termed hypercalcemia of malignancy (HCM) and is the most frequent cause of hypercalcemia in a hospital patient population. ${ }^{1-4} \mathrm{HCM}$ is a severe condition that can be fatal and occurs in $2 \%$ to $30 \%$ of adult cancer patients. ${ }^{5-9}$

$\mathrm{HCM}$ is rare in pediatric cancer patients $<18$ years of age (around $0.5 \%-1 \%$ ), and is most common in children and adolescents with leukemia, hepatocellular carcinoma, ovarian cancer, and dysgerminomas. ${ }^{4,10}$ The prevalence of pediatric HCM is not well defined. Previously, we conducted a study to estimate the prevalence of HCM among adult patients in the Clinical Practice Research Datalink (CPRD), ${ }^{11}$ a populationbased data source comprising electronic health records with laboratory data on patients in the UK. Among 37,442 adult cancer patients in 2003-2012, the prevalence of HCM overall (grade 1 or higher) increased from $0.20 \%$ to $0.67 \%$ over the study period. ${ }^{12}$ In this study, we provide a complement to the previous study by estimating the prevalence of $\mathrm{HCM}$ specifically among pediatric patients with cancer.

\section{Methods}

\section{Data resource and study population}

Data for this study were derived from the CPRD, which is housed and organized for research purposes at the Boston Collaborative Drug Surveillance Program. Briefly, the CPRD is an ongoing longitudinal database that has collected data from over 500 general practices in the UK since 1988. At the time of this study, the CPRD contained information on more than 8 million patients, of which 3 million were currently active, with a cumulative follow-up time of more than 32 million person years. It covers approximately $6 \%$ of the UK population and has a representative age and sex distribution of the entire UK population. The information contained in electronic health records includes patient demographics and characteristics, clinical diagnoses, drug prescriptions, consultant referrals, hospitalizations, and lab test results. All data accessed are de-identified and do not require patient consent for use.

We identified all patients in the CPRD younger than 18 years old who had a diagnosis of cancer (except for nonmelanoma skin cancers) between 2003 and 2014. Patients were required to have at least 1 recorded calcium and albumin laboratory value on the same day or 1 corrected serum calcium (CSC) value recorded after the cancer diagnosis. Study patients were also required to have at least 1 year of history before the cancer diagnosis to identify incident HCM cases. Patients with either a history of diagnosed hypercalcemia or hypercalcemia based on a CSC $\geq 10.8$ $\mathrm{mg} / \mathrm{dL}$ from lab data ${ }^{13,14}$ recorded more than 30 days prior to the cancer diagnosis were excluded $(0.2 \%$ of the study population), while patients who experienced HCM within 30 days before the cancer diagnosis were included. The study methods were described in more detail in a previous publication of adult HCM. ${ }^{12}$ This study was approved by the Independent Scientific Advisory Committee for UK Medicines and Healthcare products Regulatory Agency database research (protocol no: 14_031Mn).

\section{Hypercalcemia}

Hypercalcemia was defined according to four grades of CSC (10.8-11.5, 11.6-12.5, 12.6-13.5, and >13.5 mg/dL), ${ }^{13,14}$ where we used the lab-calculated CSC values (normal range 9.1-10.7). We set the upper limit of normal to $10.8 \mathrm{mg} / \mathrm{dL}$, a cutoff point commonly used to ascertain complete response to therapy. ${ }^{13}$ There were some instances $(\sim 11 \%)$ where serum calcium plus serum albumin values were the only values present. In these instances, we calculated CSC values based on the formula: $\mathrm{CSC}=0.8 \times(4-$ serum albumin $)+$ serum calcium, to determine presence of hypercalcemia. We used the highest CSC value recorded within each calendar year, where available, when estimating annual prevalence. In addition to defining HCM according to lab values, we searched the database for a recorded Read diagnosis of HCM.

\section{Statistical analysis}

From among the pediatric cancer patient population, we identified individuals who had at least one CSC value recorded within 30 days before, on, or at any time after the date of the first cancer diagnosis. Patients with data in each year from 2003 through 2014 populated the denominators for the annual prevalence. We estimated prevalence and $95 \%$ confidence intervals for each year by including patients in the numerator if they had a high CSC $(\geq 10.8 \mathrm{mg} / \mathrm{dL})$ in that calendar year using Byar's method. ${ }^{15}$ For each year that there were no calcium data in the patient record and the patient was active in the CPRD, we assumed that the CSC was normal.

\section{Results Study population}

There were 483 patients $<18$ years old in the CPRD who had a first cancer diagnosis during the years 2003 through 2014 and who had at least one CSC value recorded in the data within 30 days before, on, or after the day of the first cancer diagnosis. The median number of CSC test results during follow-up was 3 (interquartile range 1-7). The age and sex distribution of these patients, and year and type of cancer 
Table I Age, sex, calendar year, and cancer type distribution of the pediatric cancer population with CSC value in CPRD 200320 I4 ( $\mathrm{N}=483)$

\begin{tabular}{|c|c|c|c|}
\hline Characteristics & $\begin{array}{l}\text { Males } \\
\mathrm{N}=21 \text { I (\%) }\end{array}$ & $\begin{array}{l}\text { Females } \\
N=272 \text { (\%) }\end{array}$ & $\begin{array}{l}\text { Total } \\
\mathrm{N}=483 \text { (\%) }\end{array}$ \\
\hline \multicolumn{4}{|c|}{ Age at first cancer diagnosis (years) } \\
\hline$<2$ & $12(5.69)$ & $14(5.15)$ & $26(5.4)$ \\
\hline $2-<6$ & $36(17.06)$ & $23(8.46)$ & $59(12.22)$ \\
\hline $6-<12$ & $47(22.27)$ & $65(23.90)$ & $112(23.19)$ \\
\hline $12-<18$ & $116(54.98)$ & $170(62.50)$ & $286(59.21)$ \\
\hline \multicolumn{4}{|c|}{ Year of first cancer diagnosis } \\
\hline 2003 & $21(9.95)$ & $40(|4.7|)$ & $61(12.63)$ \\
\hline 2004 & $16(7.58)$ & $46(16.91)$ & $62(12.84)$ \\
\hline 2005 & $24(11.37)$ & $24(8.82)$ & $48(9.94)$ \\
\hline 2006 & $29(13.74)$ & $28(10.29)$ & $57(11.80)$ \\
\hline 2007 & $22(10.43)$ & $28(10.29)$ & $50(10.35)$ \\
\hline 2008 & $26(12.32)$ & $21(7.72)$ & $47(9.73)$ \\
\hline 2009 & $28(13.27)$ & $24(8.82)$ & $52(10.77)$ \\
\hline 2010 & $\mathrm{II}(5.2 \mathrm{I})$ & $17(6.25)$ & $28(5.80)$ \\
\hline 2011 & II (5.2I) & $19(6.99)$ & $30(6.21)$ \\
\hline 2012 & $7(3.32)$ & $9(3.31)$ & $16(3.31)$ \\
\hline 2013 & II (5.2I) & II (4.04) & $22(4.55)$ \\
\hline 2014 & $5(2.37)$ & $5(1.84)$ & $10(2.07)$ \\
\hline \multicolumn{4}{|c|}{ Cancer type at first diagnosis } \\
\hline Leukemia & $43(20.38)$ & $49(18.01)$ & $92(19.05)$ \\
\hline Lymphoma & $39(18.48)$ & $35(12.87)$ & $74(15.32)$ \\
\hline Brain tumors & $22(10.43)$ & $35(12.87)$ & $57(11.80)$ \\
\hline $\begin{array}{l}\text { Osteosarcoma and bone } \\
\text { cancers }\end{array}$ & $\mathrm{II}(5.2 \mathrm{I})$ & $19(6.99)$ & $30(6.2 I)$ \\
\hline Thyroid cancers & $--^{\mathrm{a}}$ & $-{ }^{a}$ & $18(3.73)$ \\
\hline Fibrous histiocytoma & $-^{\mathrm{a}}$ & $-^{\mathrm{a}}$ & $16(3.31)$ \\
\hline Other cancers ${ }^{\mathrm{b}}$ & $90(42.65)$ & $106(38.97)$ & $196(40.58)$ \\
\hline
\end{tabular}

Notes: aNot reported by sex owing to small cell sizes. 'Includes unknown site. Abbreviations: CSC, corrected serum calcium; CPRD, Clinical Practice Research Datalink.

diagnosis are provided in Table 1. More patients were female $(56 \%)$ and between ages 12 and 18 (59\%). Leukemia (19\%), lymphoma (15\%), and brain tumors (12\%) were the most frequent cancer types. In addition, $30(6 \%)$ had bone cancer (including osteosarcoma and Ewing sarcoma), 18 (4\%) had malignant cancer of the thyroid, and $16(3 \%)$ had fibrous histiocytomas. There were 11 cases $(2 \%)$ with neuroblastoma and $10(2 \%)$ with rhabdomyosarcoma.

\section{Prevalence of hypercalcemia}

We provide the annual prevalence of hypercalcemia by CSC grade level in Table 2 below. There were 6 children out of 483 who had a high CSC value recorded. Cancers included endocrine adenoma, Wilms tumor, acute lymphoid leukemia, malignant cancer of the thyroid, and cancer of the tongue.

Based on CSC lab values, we estimated that $0.26 \%$ $0.88 \%$ of pediatric cancer patients were affected by HCM in 2003-2014. We did not find additional HCM cases when we searched on Read codes for hypercalcemia.
Table 2 Annual prevalence (\%) of HCM among 483 pediatric cancer patients in the CPRD 2003-2014

\begin{tabular}{llll}
\hline Year & $\begin{array}{l}\text { Number of cancer } \\
\text { patients }\end{array}$ & $\begin{array}{l}\text { Grade I or higher: } \\
\text { CSC } \geq \mathbf{I 0 . 8}\end{array}$ \\
\cline { 3 - 4 } & & $\begin{array}{l}\text { HCM } \\
\text { cases }\end{array}$ & $\begin{array}{l}\text { Prevalence } \\
(95 \% ~ C l)\end{array}$ \\
\hline 2003 & 61 & 0 & $\mathrm{n} / \mathrm{a}$ \\
2004 & 122 & 0 & $\mathrm{n} / \mathrm{a}$ \\
2005 & 170 & 0 & $\mathrm{n} / \mathrm{a}$ \\
2006 & 226 & $\mathrm{NR}^{\mathrm{d}}$ & $0.88^{\mathrm{a}}$ \\
& & & $(0.11-3.16)$ \\
2007 & 272 & 0 & $\mathrm{n} / \mathrm{a}$ \\
2008 & 310 & $\mathrm{NR}^{\mathrm{d}}$ & $0.65^{\mathrm{a}}$ \\
& & & $(0-2.31)$ \\
2009 & 354 & $\mathrm{NR}^{\mathrm{d}}$ & $0.28^{\mathrm{b}}$ \\
& & & $(0-1.56)$ \\
2010 & 374 & 0 & $\mathrm{n} / \mathrm{a}$ \\
2011 & 386 & 0 & $\mathrm{n} / \mathrm{a}$ \\
2012 & 384 & $\mathrm{NR}^{\mathrm{d}}$ & $0.26^{\mathrm{b}}$ \\
& & & $(0-1.44)$ \\
2013 & 371 & $N^{d}$ & $0.27^{\mathrm{c}}$ \\
& & & $(0-1.49)$ \\
2014 & 324 & 0 & $\mathrm{n} / \mathrm{a}$ \\
\hline
\end{tabular}

Notes: Patients had HCM grades ${ }^{a} 1=10.8 \leq \operatorname{CSC} \leq 11.5,{ }^{b} 2=11.5<\operatorname{CSC} \leq 12.5$, ${ }^{c} 3=12.5<\mathrm{CSC} \leq 13.5$. ${ }^{d} \mathrm{NR}$, not reported (owing to small cell size).

Abbreviations: HCM, hypercalcemia of malignancy; CPRD, Clinical Practice Research Datalink; CSC, corrected serum calcium; n/a, not applicable.

\section{Discussion}

In this population of 483 pediatric patients with cancer during the years 2003-2014 in the UK CPRD, the prevalence of HCM was low for all years, consistent with the rates estimated in previous publications. ${ }^{10,16,17} \mathrm{We}$ estimated that the prevalence of grade $1 \mathrm{HCM}$ ranged from $0.65 \%$ to $0.88 \%$ (data not shown) and the prevalence of HCM overall (grade 1 or higher) ranged from $0.26 \%$ to $0.88 \%$ between 2003 and 2014. There were only 483 pediatric cancer patients for whom a CSC value was available in the database, and only 6 of these had identified HCM, and thus the numbers were small.

Hypercalcemia is a serious metabolic complication of malignancy that is less common in children than in adults. ${ }^{10}$ A single institutional retrospective study carried out by McKay and Furman ${ }^{16}$ in the USA found that only $0.4 \%$ of all children treated for cancer at their hospital over a 29-year period developed HCM. Moayeri et $\mathrm{al}^{17}$ reported a higher prevalence: $5.4 \%$ of children hospitalized with cancer ( 8 out of 148) had HCM with half of the cases associated with leukemia. Different from the McKay and Moayeri studies, our study was not restricted to children hospitalized with cancer and provides estimates of prevalence on a population-based cohort of children diagnosed with cancer. This study includes data on all individuals registered with selected general practitioners, and thus includes HCM cases identified from a 
general patient population rather than a hospital or registry population. Further, the cutoffs for HCM used by McKay and Moayeri were different from those used in this study, so it is possible that our results are not directly comparable to the results of these earlier studies.

While the population-based nature of this study is a strength, the study also has several limitations. These results were limited to patients who had recorded laboratory values for serum calcium and serum albumin (or CSC), and thus we may have missed some patients with elevations in calcium since not all patients with cancer had details of cancer complications recorded in their GP record. In addition, only 483 (22\%) out of 2,189 pediatric cancer patients had at least one test for serum calcium and albumin (or CSC) and were therefore eligible for inclusion in the study. It is possible that in cancer patients, GPs do not routinely record individual cancer-related complications, in particular those based on hospital findings. Also, laboratory calcium testing is more likely to be carried out for patients who are suspected to be hypercalcemic, although the extent of this nonrandom testing is not known.

Our study provides an estimate of HCM prevalence across patients of all stages; it is likely that patients with more advanced cancer have higher prevalence of HCM. However, we were unable to stratify by stage owing to limited sample size, which would have been further reduced when restricting to patients with stage data available in the CPRD. Further, values were not recorded in each calendar year, and thus we did not always know when CSC values returned to normal. Finally, the CPRD only provides year of birth for confidentiality reasons, and therefore we calculated patient's age assuming their birth date was July 1 in the year of their birth. Thus, we may have misclassified some young adults as children, or vice versa.

\section{Conclusion}

These data provide information on a large number of pediatric cancer patients with at least one lab CSC value in a population-based data resource. The prevalence of HCM was low and is consistent with the finding that HCM is rare in cancer patients under age 18 .

\section{Author contributions}

S Jick participated in the conception and design of the study, and contributed to data analysis. L Li contributed to study design and conducted the analyses. VM Gastanaga, A Liede, and RK Hernandez participated in the conception and design of the study, and contributed to data analysis and interpretation. All the authors participated in revisions of the manuscript for intellectual content. S Jick is the guarantor of this work and, as such, had full access to all the data in the study and takes responsibility for the integrity of the data and the accuracy of the data analysis. All the authors contributed to study concept, design, and interpretation, manuscript preparation, critical editing, and review, gave final approval of the version to be published, and agree to be accountable for all aspects of the work.

\section{Disclosure}

This study was funded by Amgen Inc. The coauthors VMG, $\mathrm{AL}$, and RKH are employees of Amgen Inc. The authors report no other conflicts of interest in this work.

\section{References}

1. Rosner MH, Dalkin AC. Onco-nephrology: the pathophysiology and treatment of malignancy-associated hypercalcemia. Clin J Am Soc Nephrol. 2012;7(10):1722-1729.

2. Strewler GJ, Nissenson RA. Hypercalcemia in malignancy. West JMed. 1990;153(6):635-640.

3. Martin TJ. Hypercalcemia of malignancy. Epidemiology and pathogenesis. Clin Rev Bone Miner Metab. 2002;1(1):51-63.

4. Minisola S, Pepe J, Piemonte S, Cipriani C. The diagnosis and management of hypercalcaemia. BMJ. 2015;350:h2723.

5. Grill V, Martin TJ. Hypercalcemia of malignancy. Rev Endocr Metab Disord. 2000;1(4):253-263.

6. Body JJ. Hypercalcemia of malignancy. Sem Nephrol. 2004;24(1):48-54.

7. Basso U, Maruzzo M, Roma A, Camozzi V, Luisetto G, Lumachi F. Malignant hypercalcemia. Curr Med Chem. 2011;18:3462-3467.

8. Lumachi F, Brunello A, Roma A, Basso U. Cancer-induced hypercalcemia. Anticancer Res. 2009;29(5):1551-1555.

9. Gastanaga VM, Schwartzberg LS, Jain RK, et al. Prevalence of hypercalcemia among cancer patients in the United States. Cancer Med. 2016;5(8):2091-2100.

10. Lietman SA, Germain-Lee EL, Levine MA. Hypercalcemia in children and adolescents. Curr Opin Pediatr. 2010;22(4):508-515.

11. National Institute for Health Research and the Medicines and Healthcare products Regulatory Agency [homepage on the Internet]. Available from: http://www.cprd.com/home/. Accessed June 1, 2014.

12. Jick S, Li L, Gastanaga VM, Liede A. Prevalence of hypercalcemia of malignancy among cancer patients in the UK: analysis of the Clinical Practice Research Datalink Database. Cancer Epidemiol. 2015;39(6):901-907.

13. Hu MI, Glezerman IG, Leboulleux S, et al. Denosumab for treatment of hypercalcemia of malignancy. J Clin Endocrinol Metab. 2014; 99(9):3144-3152.

14. Cancer Therapy Evaluation Program, Common Terminology Criteria for Adverse Events (CTCAE), Version 3.0. Available from: http://ctep. cancer.gov/protocoldevelopment/electronic_applications/docs/ctcaev3. pdf. Accessed January 31, 2014.

15. Rothman KJ, Boice JD Jr. Epidemiologic Analysis with a Programmable Calculator (NIH Publication 79-1649). Washington, DC: US Government Printing Office; 1979.

16. McKay C, Furman WL. Hypercalcemia complicating childhood malignancies. Cancer. 1993;72(1):256-260.

17. Moayeri H, Oloomi Z, Sambo SA. A cross-sectional study to determine the prevalence of calcium metabolic disorder in malignant childhood cancers in patients admitted to the pediatric ward of Vali-Asr Hospital. Acta Med Iran. 2011;49(12):818-823. 
Clinical Epidemiology

\section{Publish your work in this journal}

Clinical Epidemiology is an international, peer-reviewed, open access, online journal focusing on disease and drug epidemiology, identification of risk factors and screening procedures to develop optimal preventative initiatives and programs. Specific topics include: diagnosis, prognosis, treatment, screening, prevention, risk factor modification,

Submit your manuscript here: https://www.dovepress.com/clinical-epidemiology-journal
Dovepress

systematic reviews, risk and safety of medical interventions, epidemiology and biostatistical methods, and evaluation of guidelines, translational medicine, health policies and economic evaluations. The manuscript management system is completely online and includes a very quick and fair peer-review system, which is all easy to use. 